RESENDE, P. C.; CAMPOS, G. L. Estudo sobre as principais regulamentações dos campos eletromagnéticos em linhas de transmissão operando em regime permanente

\title{
ESTUDO SOBRE AS PRINCIPAIS REGULAMENTAÇÕES DOS CAMPOS ELETROMAGNÉTICOS EM LINHAS DE TRANSMISSÃO OPERANDO EM REGIME PERMANENTE ${ }^{1}$
}

\author{
Paula Carvalho Resende ${ }^{2}$ \\ Gustavo Lobato Campos ${ }^{3}$ \\ Mariana Guimarães dos Santos ${ }^{4}$
}

\begin{abstract}
RESUMO
Este artigo tem como objetivo apresentar, de modo geral, as principais regulamentações e recomendações nacionais e internacionais, referentes aos níveis de limite de exposição humana a campos elétricos e magnéticos para linhas de transmissão, operando na frequência de 50-60 Hz. Para tal, realiza-se uma pesquisa para identificar as principais instituições nacionais e internacionais credenciadas para elaborar as regulamentações. Após este estudo, são feitos resumos das normas regulamentadoras sobre campos eletromagnéticos de cada instituição, a fim de facilitar o entendimento do leitor e mostrar de forma simplificada os limites de campos elétricos e magnéticos estabelecidos pelas instituições.
\end{abstract}

Palavras Chave: Linhas de transmissão. Campos elétricos. Campos magnéticos. Regulamentação. Nível de referência.

\section{INTRODUÇÃO}

As linhas de transmissão de energia elétrica são utilizadas para transportar a energia dos pontos de geração para os centros consumidores. A predominância da hidroeletricidade na matriz energética nacional, com bacias geograficamente distantes entre si, faz com que as linhas de transmissão exerçam um papel importante, interligando o centro gerador as demais localidades consumidoras no país (CAMPOS, 2010).

Devido a problemas geográficos, econômicos ou sociais algumas linhas de transmissão são construídas próximas a áreas residenciais, comerciais ou industriais, causando

\footnotetext{
${ }^{1}$ Como citar este artigo: RESENDE, C. P.; CAMPOS, L. G. Estudo sobre as principais regulamentações dos campos eletromagnéticos em linhas de transmissão operando em regime permanente. ForScience: revista científica do IFMG, Formiga, v. 5, n. 3, e00215, jul./dez. 2017.

${ }^{2}$ Mestranda em Engenharia Elétrica pelo Centro Federal de Educação Tecnológica de Minas Gerais (CEFETMG). E-mail: pcarvalhoresende@gmail.com. Currículo Lattes: http://lattes.cnpq.br/4425879041210715.

${ }^{3}$ Doutor em Engenharia Nuclear pela Universidade Federal de Minas Gerais (UFMG) e professor efetivo do Instituto Federal de Minas Gerais (IFMG - Campus Formiga). E-mail: gustavo.lobato@ifmg.edu.br. Currículo Lattes: http://lattes.cnpq.br/9613750934178733.

${ }^{4}$ Mestra em Engenharia Elétrica pela Universidade Federal de São João Del Rei (UFSJ) e professora efetiva do (IFMG - Campus Formiga). E-mail.: mariana.santos@ifmg.edu.br. Currículo Lattes: http://lattes.cnpq.br/466159 8896242893.
} 
RESENDE, P. C.; CAMPOS, G. L. Estudo sobre as principais regulamentações dos campos eletromagnéticos em linhas de transmissão operando em regime permanente

na comunidade local certa preocupação em relação às interferências em sistemas eletrônicos e aos efeitos biológicos quanto à exposição a campos elétricos e magnéticos (COSTA; MONTEIRO).

Por isso, as concessionárias de energia elétrica têm mostrado grande interesse em se adequar aos valores limite de campos elétricos e magnéticos estabelecidos pelas instituições nacionais e internacionais credenciadas na ampliação do sistema elétrico (BECKER, 2008).

Dessa forma, este artigo tem como finalidade apresentar um estudo das principais regulamentações internacionais e as regulamentações vigentes no Brasil, referentes aos níveis de campo elétrico e magnético em regime permanente, ou seja, operando em $50 \mathrm{~Hz}$ a $60 \mathrm{~Hz}$ emitidos por linhas de transmissão para público em geral e ocupacional.

\section{REFERENCIAL TEÓRICO}

\subsection{Conceitos básicos de linhas de transmissão}

As linhas de transmissão são responsáveis pela transmissão de energia elétrica do Sistema Elétrico de Potência brasileiro que também é composto pela geração, distribuição e comercialização de energia. Importante ainda citar que devido ao contínuo crescimento dos sistemas elétricos mundiais, imposto pela crescente demanda de energia elétrica, o tópico sobre linhas de transmissão são objetos de estudos ao longo dos últimos anos.

Cabos fase, cabos para-raios, torres, cadeias de isoladores, ferragens e aterramentos elétricos são componentes básicos das linhas de transmissão. Elas podem operar em regime permanente, com frequências de $50 \mathrm{~Hz}$ a $60 \mathrm{~Hz}$, foco do trabalho, ou em regime transitório, em que as frequências podem chegar até $10 \mathrm{MHz}$, causadas por operações de chaveamentos, curto circuito ou descargas atmosféricas.

No território brasileiro os níveis de tensão das linhas são diferenciados devido a parâmetros físicos e econômicos. A elevação de tensão das linhas de transmissão, eleva os níveis de corrente elétrica e, por consequência, eleva os níveis de interferência eletromagnética nas suas faixas de passagens, que podem ser nocivos às instalações comerciais e residenciais e seres vivos localizados dentro da faixa (VIEIRA, 2013).

Portanto, há uma enorme gama de regulamentações para a construção e operação de linhas de transmissão, que é de extrema importância para o setor elétrico. Esse artigo faz o estudo das normas referentes aos campos eletromagnéticos, apresentadas nos tópicos a seguir, 
RESENDE, P. C.; CAMPOS, G. L. Estudo sobre as principais regulamentações dos campos eletromagnéticos em linhas de transmissão operando em regime permanente

para limitar valores de exposição a campos elétricos e magnéticos a fim de garantir segurança nas instalações próximas das linhas de transmissão.

\section{METODOLOGIA}

Inicialmente realizou-se uma pesquisa sobre as principais instituições internacionais que regulamentam sobre campos eletromagnéticos. As regulamentações internacionais mais referenciadas foram produzidas pela Comissão Internacional de Proteção Contra as Radiações Não Ionizantes (ICNIRP), pelo Instituto de Engenheiros Eletricistas e Eletrônicos (IEEE) e pelo Conselho da União Europeia. Os estudos internacionais são antigos, porém são regularmente revistos e avaliados a partir de novos conhecimentos e novos desenvolvimentos tecnológicos. As recomendações e regulamentações do ICNRIP e do Conselho da União Europeia são empregadas em alguns países da Europa que estão associados a estes núcleos. Porém, a ICNIRP é referenciada na maioria das regulamentações no mundo todo. As normas regulamentadoras do IEEE são utilizadas em alguns países da América, apesar das normas brasileiras utilizarem as três instituições como referência. Porém, as regulamentações internacionais não especificam níveis de referência à exposição de campos eletromagnéticos somente para linhas de transmissão de energia.

Os níveis de referência das instituições internacionais são para qualquer equipamento que emita campos eletromagnéticos em determinadas frequências. Por isso, os estudos das normas internacionais apresentados a seguir são das publicações das instituições em que a frequência de operação das linhas de transmissão esteja incluída, ou seja, entre $50 \mathrm{~Hz}$ a $60 \mathrm{~Hz}$.

Posteriormente, os estudos partiram para as instituições nacionais. A Associação Brasileira de Normas Técnicas (ABNT) é a principal instituição responsável pelas normatizações brasileiras. Porém, outros órgãos têm o direito de atualizar as normas brasileiras, por meio de leis e resoluções. Desta forma, com o intuito de apresentar de modo geral e simplificado as regulamentações nacionais e internacionais, foram feitos resumos das principais regulamentações sobre campos eletromagnéticos, como resultados deste artigo.

\section{RESULTADOS}


RESENDE, P. C.; CAMPOS, G. L. Estudo sobre as principais regulamentações dos campos eletromagnéticos em linhas de transmissão operando em regime permanente

\subsection{Principais regulamentações internacionais sobre campos eletromagnéticos}

\subsubsection{Comissão Internacional de Proteção Contra as Radiações Não Ionizantes - ICNIRP}

A ICNIRP (Comissão Internacional de Proteção Contra as Radiações Não Ionizantes) é uma organização independente, reconhecida mundialmente, que estabelece orientações para a exposição de seres humanos a campos elétricos e magnéticos. A ICNIRP separa seus estudos e orientações à exposição de campos por faixas de frequências, pois cada faixa tem um efeito diferente sobre a saúde humana. No presente artigo, o foco de estudo é de campos eletromagnéticos operando em regime permanente e por isso, a diretriz estudada da ICNIRP é referente a baixas frequências, entre $1 \mathrm{~Hz}$ a $100 \mathrm{kHz}$.

A publicação da ICNIRP para baixas frequências tem como objetivo estabelecer linhas de orientação visando limitar a exposição de seres humanos a campos elétricos e magnéticos de baixas frequências a fim de proteger contra os efeitos diretos e indiretos causados à saúde. Segundo esta publicação, as limitações basearam-se em evidências comprovadas relacionadas a efeitos agudos, porém não há evidências convincentes que patologias crônicas estejam relacionadas à exposição de campos eletromagnéticos de baixas frequências. Devido a estas incertezas dos dados científicos, são definidas limitações a estes campos.

No decorrer do seu texto, a publicação da ICNIRP para baixas frequências apresenta algumas unidades e grandezas com a finalidade de esclarecer ao leitor as diferenças entre campo elétrico e magnético e como eles interagem com o ambiente em sua volta. A ICNIRP também explica os mecanismos de acoplamento entre os campos eletromagnéticos e o corpo humano. A partir disso, a publicação revisa os principais estudos e conclusões das literaturas científicas atuais sobre as epidemiologias que podem estar associadas aos efeitos dos campos eletromagnéticos, como, por exemplo, doenças cardiovasculares, câncer, e entre outras.

Com base nos estudos epidemiológicos, a ICNIRP estabelece linhas de orientações para limitar a exposição a campos eletromagnéticos para frequências entre $1 \mathrm{~Hz}$ e $10 \mathrm{MHz}$ para público geral e ocupacional. Esses níveis de referência são obtidos a partir das limitações básicas através de cálculos na situação de acoplamento máximo do campo que o indivíduo está exposto. Como esta publicação utiliza uma faixa de frequência para limitar os campos, todos eles são em função da frequência. A Tabela 1 mostra os níveis de referência para exposição ocupacional a campos eletromagnéticos ao longo do tempo e a Tabela 2 mostra os 
RESENDE, P. C.; CAMPOS, G. L. Estudo sobre as principais regulamentações dos campos eletromagnéticos em linhas de transmissão operando em regime permanente

níveis de referência para o público em geral, onde $\mathrm{f}$ é a frequência em Hertz (INTERNATIONAL COMMISSION ON NON-IONIZING RADIATION PROTECTION, 2010).

Tabela 1 - Níveis de referência para exposição do público ocupacional a campos eletromagnéticos variáveis ao longo do tempo (valores rms de campo não perturbado)

\begin{tabular}{ccc}
\hline Gama de Frequências & $\begin{array}{c}\text { Intensidade do campo } \\
\text { elétrico } \mathbf{E}(\mathbf{k V / m})\end{array}$ & $\begin{array}{c}\text { Densidade de fluxo } \\
\text { magnético B (T) }\end{array}$ \\
\hline $1 \mathrm{~Hz}-8 \mathrm{~Hz}$ & 20 & $0,2 / \mathrm{f}^{2}$ \\
$8 \mathrm{~Hz}-25 \mathrm{~Hz}$ & 20 & $2,5 \times 10^{-2} / \mathrm{f}$ \\
$25 \mathrm{~Hz}-300 \mathrm{~Hz}$ & $5 \times 10^{2} / \mathrm{f}$ & $1 \times 10^{-3}$ \\
$300 \mathrm{~Hz}-3 \mathrm{kHz}$ & $5 \times 10^{2} / \mathrm{f}$ & $0,3 / \mathrm{f}$ \\
$3 \mathrm{kHz}-10 \mathrm{MHz}$ & $1,7 \times 10^{-1}$ & $1 \times 10^{-4}$ \\
\hline
\end{tabular}

Fonte: International Commission on Non-ionizing Radiation Protection (2010).

Tabela 2 - Níveis de referência para exposição do público em geral a campos eletromagnéticos variáveis ao longo do tempo (valores rms de campo não perturbado)

\begin{tabular}{ccc}
\hline Gama de Frequências & $\begin{array}{c}\text { Intensidade do campo } \\
\text { elétrico } \mathbf{E}(\mathbf{k V / m})\end{array}$ & $\begin{array}{c}\text { Densidade de fluxo } \\
\text { magnético B (T) }\end{array}$ \\
\hline $1 \mathrm{~Hz}-8 \mathrm{~Hz}$ & 5 & $4 \times 10^{-2} / \mathrm{f}^{2}$ \\
$8 \mathrm{~Hz}-25 \mathrm{~Hz}$ & 5 & $5 \times 10^{-3} / \mathrm{f}$ \\
$25 \mathrm{~Hz}-300 \mathrm{~Hz}$ & 5 & $2 \times 10^{-4}$ \\
$50 \mathrm{~Hz}-400 \mathrm{~Hz}$ & $2,5 \times 10^{2} / \mathrm{f}$ & $2 \times 10^{-4}$ \\
$400 \mathrm{~Hz}-3 \mathrm{kHz}$ & $2,5 \times 10^{2} / \mathrm{f}$ & $8 \times 10^{-2} / \mathrm{f}$ \\
$3 \mathrm{kHz}-10 \mathrm{MHz}$ & $8,3 \times 10^{-2}$ & $2,7 \times 10^{-5}$ \\
\hline
\end{tabular}

Fonte: International Commission on Non-ionizing Radiation Protection (2010).

Contudo, os valores de campos apresentados nas Tabelas 1 e 2 são para os públicos expostos bem próximos das fontes dos campos, ou seja, dentro da faixa de passagem das fontes destes campos.

Após os níveis de referência, a publicação da ICNIRP para baixas frequências apresenta as medidas que podem ser tomadas para a proteção das pessoas expostas aos campos eletromagnéticos, principalmente para o público ocupacional, utilizando um vestuário adequado, luvas isoladas, entre outros equipamentos de segurança pessoal (INTERNATIONAL COMMISSION ON NON-IONIZING RADIATION PROTECTION, 2010). 
RESENDE, P. C.; CAMPOS, G. L. Estudo sobre as principais regulamentações dos campos eletromagnéticos em linhas de transmissão operando em regime permanente

\subsubsection{Instituto de Engenheiros Eletricistas e Eletrônicos - IEEE}

O IEEE (Instituto de Engenheiros Eletricistas e Eletrônicos) é um órgão profissional sem fins lucrativos fundado nos Estados Unidos dedicado ao avanço da tecnologia para benefício da humanidade. Por isso, o instituto realiza estudos, publicações e conferências com a finalidade do desenvolvimento, integração, compartilhamento e o conhecimento aplicado no que se refere à ciência e tecnologias da eletricidade e da informação (INSTITUTE OF ELECTRIC AND ELECTRONIC ENGINEERS - ADVANCING TECHNOLOGY FOR HUMANITY, [20--]). Assim, pensando na segurança da humanidade referente à exposição dos campos eletromagnéticos, o IEEE elaborou o documento IEEE Std C95.6 de 2002 para padronizar os níveis de exposição humana a campos eletromagnéticos para frequências entre $0-3 \mathrm{kHz}$.

Esse documento, como as demais normas regulamentadoras, apresenta no decorrer do seu texto algumas definições, termos e símbolos para facilitar o entendimento do leitor, úteis para a aplicação da norma. Após as definições, o documento mostra as medidas de proteção que podem ser adotadas para prevenir ou minimizar a exposição a campos eletromagnéticos e ainda apresenta os mecanismos de reações físicas e os efeitos biológicos adversos que os seres humanos estão expostos, como excitação cardíaca ou muscular, entre outros.

Para apresentar os níveis de referência para exposição de campos elétricos e magnéticos, a norma C95.6 relaciona a exposição de algumas partes do corpo humano e diferencia seus níveis de referência. A Tabela 3 mostra os níveis máximos de exposição permitidos para o público ocupacional. A Tabela 4 mostra os níveis máximos de exposição permitidos para o público em geral (INSTITUTE OF ELECTRIC AND ELECTRONIC ENGINEERS - INTERNACIONAL COMMITTEE ON ELECTROMAGNETIC SAFETY, 2006).

Tabela 3 - Níveis de referência para exposição do público ocupacional a campos eletromagnéticos variáveis ao longo do tempo nas frequências de $0-3 \mathrm{kHz}$ (valores rms de campo não perturbado)

\begin{tabular}{cccc}
\hline Campos & $\begin{array}{c}\text { Intensidade do campo } \\
\text { elétrico }(\mathbf{k V} / \mathbf{m})\end{array}$ & $\begin{array}{c}\text { Densidade de fluxo magnético } \\
(\mathbf{m T})\end{array}$ \\
\hline Parte do Corpo & Corpo todo & Cabeça & Braços e Pernas \\
Valor & 20 & 2,71 & 63,2 \\
\hline
\end{tabular}

Fonte: Institute of Electric and Electronic Engineers - Advancing Technology for Humanity ([20--]). ForSci.: r. cient. IFMG, Formiga, v. 5, n. 3, e00215, jul./dez 2017. 
RESENDE, P. C.; CAMPOS, G. L. Estudo sobre as principais regulamentações dos campos eletromagnéticos em linhas de transmissão operando em regime permanente

Tabela 4 - Níveis de referência para exposição do público em geral a campos eletromagnéticos variáveis ao longo do tempo nas frequências de $0-3 \mathrm{kHz}$ (valores rms de campo não perturbado)

\begin{tabular}{cccc}
\hline Campos & $\begin{array}{c}\text { Intensidade do campo } \\
\text { elétrico }(\mathbf{k V} / \mathbf{m})\end{array}$ & $\begin{array}{c}\text { Densidade de fluxo magnético } \\
(\mathbf{m T})\end{array}$ \\
\hline Parte do Corpo & Corpo todo & Cabeça & Braços e Pernas \\
Valor & 10 & 0,904 & 63,2 \\
\hline
\end{tabular}

Fonte: Institute of Electric and Electronic Engineers - Advancing Technology for Humanity ([20--]).

Após apresentar os níveis máximos de referência a exposição humana, a norma detalha a metodologia utilizada no seu desenvolvimento, mostrando os estudos bibliográficos empregados para chegar aos níveis de referência mostrados acima (INSTITUTE OF ELECTRIC AND ELECTRONIC ENGINEERS - INTERNACIONAL COMMITTEE ON ELECTROMAGNETIC SAFETY, 2006).

\subsubsection{Conselho da União Europeia}

O Conselho Europeu ou União Europeia (UE) é uma instituição que define as orientações e prioridades políticas gerais da União Europeia. O Conselho Europeu é composto pelos Chefes de Estado ou de Governo dos Estados-Membros, bem como pelo seu Presidente e pelo Presidente da Comissão (EUROPEAN UNION, 2016). A recomendação do Conselho resulta no processo de negociação entre o Conselho e o Parlamento Europeu. Por isso, a maioria das decisões tomadas pelo conselho se aplica à legislação da União Europeia.

A publicação e recomendação do Conselho Europeu sobre exposição a campos eletromagnéticos para frequências entre $0 \mathrm{~Hz}$ a $300 \mathrm{GHz}$ foi publicada em julho de 1999. No caso desse documento, o parlamento europeu convidou o Conselho Europeu para propor medidas legislativas para limitar a exposição do público ocupacional e geral a campos eletromagnéticos.

Essa medida legislativa tem como objetivo a proteção da saúde do público e se aplica às pessoas que estejam expostas tempo significativo aos campos eletromagnéticos. Ela se baseia nas documentações científicas existentes e nas recomendações publicadas pela ICNIRP. 
RESENDE, P. C.; CAMPOS, G. L. Estudo sobre as principais regulamentações dos campos eletromagnéticos em linhas de transmissão operando em regime permanente

Como as demais normas regulamentadoras, algumas definições são estabelecidas para o entendimento técnico da mesma. A regulamentação define algumas restrições básicas, pois dependendo da frequência exposta e o tempo de exposição, os efeitos à saúde humana podem ser evitados.

Os níveis de referência para limitar a exposição são obtidos a partir da condição de acoplamento máximo do campo para o indivíduo exposto, proporcionando assim uma proteção máxima. A Tabela 5 mostra os valores de referência para campos elétricos e magnéticos recomendados para exposição do público em geral e ocupacional. Como esta publicação utiliza uma faixa de frequência para limitar os campos, os valores estão em função de f, ou seja, a frequência (THE COUNCIL OF THE EUROPEAN UNION, 1999).

Tabela 5 - Níveis de referência para exposição do público em geral e ocupacional a campos elétricos e magnéticos variáveis ao longo do tempo (valores rms de campo não perturbado)

\begin{tabular}{ccc}
\hline Gama de Frequências & $\begin{array}{c}\text { Intensidade do campo } \\
\text { elétrico } \mathbf{E}(\mathbf{V} / \mathbf{m})\end{array}$ & $\begin{array}{c}\text { Densidade de fluxo } \\
\text { magnético B }(\boldsymbol{\mu} \mathbf{T})\end{array}$ \\
\hline $0 \mathrm{~Hz}-1 \mathrm{~Hz}$ & -- & $4 \times 10^{4}$ \\
$1 \mathrm{~Hz}-8 \mathrm{~Hz}$ & 10000 & $4 \times 10^{4} / \mathrm{f}^{2}$ \\
$8 \mathrm{~Hz}-25 \mathrm{~Hz}$ & 10000 & $5000 / \mathrm{f}$ \\
$25 \mathrm{~Hz}-80 \mathrm{~Hz}$ & $250 / \mathrm{f}$ & $5 / \mathrm{f}$ \\
$80 \mathrm{~Hz}-3 \mathrm{kHz}$ & $250 / \mathrm{f}$ & 6,25 \\
$3 \mathrm{kHz}-150 \mathrm{kHz}$ & 87 & 6,25 \\
$150 \mathrm{kHz}-1 \mathrm{MHz}$ & 87 & $0,92 / \mathrm{f}$ \\
$1 \mathrm{MHz}-10 \mathrm{MHz}$ & $87 / \mathrm{f}^{1 / 2}$ & $0,92 / \mathrm{f}$ \\
$10 \mathrm{MHz}-0,4 \mathrm{GHz}$ & 28 & 0,092 \\
$0,4 \mathrm{GHz}-2 \mathrm{GHz}$ & $1,375 \mathrm{f}^{1 / 2}$ & $0,0046 \mathrm{f}^{1 / 2}$ \\
$2 \mathrm{GHz}-300 \mathrm{GHz}$ & 61 & 0,20 \\
\hline
\end{tabular}

Fonte: The Cuncil of the European Union (1999).

Apesar das normas internacionais apresentarem várias faixas de frequências para avaliar os valores de campos, pode-se observar que os valores em que se adequa a faixa de frequência de uma linha de transmissão não variam muito, devido ao fato da maioria das regulamentações utilizarem as mesmas bases científicas. 
RESENDE, P. C.; CAMPOS, G. L. Estudo sobre as principais regulamentações dos campos eletromagnéticos em linhas de transmissão operando em regime permanente

É importante exibir os conceitos básicos das principais normas internacionais, para que fique claro para o leitor a origem dos níveis de referências utilizados nas normas brasileiras empregadas atualmente.

\subsection{Regulamentações brasileiras sobre campos eletromagnéticos na operação em regime permanente de linhas de transmissão}

\subsubsection{Projeto de Linhas Aéreas de Transmissão de Energia Elétrica - NBR 5422}

A norma NBR 5422 de fevereiro de 1985, atualmente em processo de revisão (SOUZA, 2012), estabelece as condições básicas para projeto, reisolamento e/ou reforma de linhas de transmissão aéreas com valores eficazes fase-fase de tensão máxima de $38 \mathrm{kV}$ até $800 \mathrm{kV}$, a fim de garantir níveis mínimos de segurança e limitar perturbações em instalações próximas. No entanto, as instalações provisórias não precisam, necessariamente, atender às prescrições estabelecidas pela NBR 5422.

Para entender os termos técnicos no decorrer do seu texto, a NBR 5422 apresenta algumas terminologias importantes como a definição de vão de vento e os principais símbolos utilizados, entre outros. Além das definições, também são apresentados alguns parâmetros meteorológicos e correções de coeficientes, como temperatura máxima e mínima, correções de rugosidades, correções de altura, dentre outros.

$\mathrm{Na}$ descrição dos materiais, os cabos condutores e cabos para-raios devem atender prescrições estabelecidas pela NBR 5422 e também outras normas mais específicas. A NBR 5422 constam algumas condições ambientais para os cabos e condutores, como as temperaturas médias, considerando ação do vento ou não, para cálculo mecânico da flecha máxima, por exemplo. Os cabos e condutores também devem utilizar dispositivos especiais de proteção contra danos devido à vibração eólica, principalmente em terrenos planos, travessias de grandes rios, entre outros.

Assim como os cabos e condutores, os isoladores e ferragens e os suportes e fundações também devem obedecer a outras normas brasileiras mais específicas. Porém, a NBR 5422 especifica que os isoladores não devem ser submetidos a esforços superiores a $40 \%$ do nominal. Os suportes devem ser projetados para cargas consideradas no projeto, como isoladores, ventos, etc. Ela determina as características dos esforços que o suporte está sujeito, 
RESENDE, P. C.; CAMPOS, G. L. Estudo sobre as principais regulamentações dos campos eletromagnéticos em linhas de transmissão operando em regime permanente

divididos em três tipos: cargas de ventos, cargas permanentes e cargas especiais. As fundações devem ser feitas de maneira a garantir maior estabilidade dos suportes, com materiais resistentes à corrosão.

De acordo com a NBR 5422, distância de segurança é a distância mínima de afastamento dos condutores e seus acessórios da própria linha de transmissão, do terreno ou obstáculos atravessados pela mesma. Ela estabelece condições de operação e parâmetros para cálculo da distância mínima do suporte, da distância mínima do condutor ao solo ou aos obstáculos em condições normais de operação ou emergência e da distância mínima entre condutores em suportes diferentes.

Em relação ao aterramento, os suportes da linha de transmissão devem ser aterrados de modo que não interfira nas instalações dentro ou próximas às faixas de segurança, oferecendo bom desempenho e segurança. O método de aterramento fica a critério do proprietário da linha. Porém, os materiais devem ser resistentes à corrosão e com durabilidade compatível com a vida útil da linha de transmissão.

Para os projetos de travessias, a NBR 5422 determina as condições que devem ser satisfeitas na construção de linhas sobre outras linhas elétricas, ou de telecomunicações, vias de transporte, florestas e demais formas de vegetação, rios e lagos, etc. Também, deve ser avaliada a influência eletromagnética na faixa da travessia.

Conforme estabelecido pela NBR 5422, a faixa de segurança de uma linha de transmissão deve ser determinada levando-se em conta o balanço dos cabos devido à ação do vento, efeitos elétricos e posicionamento das fundações de suportes e estais. Esses aspectos definirão a escolha da largura da faixa de passagem. Para tensão superior a $230 \mathrm{kV}$ deve ser verificado o nível de interferências eletromagnéticas para a determinação da faixa de segurança. Destaca-se que o valor de campo elétrico ao nível do solo não deve ultrapassar 5 $\mathrm{kV} / \mathrm{m}$ na faixa e recomenda-se que os estais fiquem situados dentro da mesma.

Onde for necessário, deverá ser feito na faixa de construção da linha de transmissão, uma limpeza para implantação, operação e manutenção da linha, condizendo com as leis ambientais e com menor impacto possível.

As linhas próximas de aeroportos devem ser projetadas a fim de ficarem abaixo do gabarito de aproximação do aeroporto e ser sinalizada, conforme o decreto $\mathrm{n}^{\circ} 83399$ de 03/05/79 (ASSOCIAÇÃO BRASILEIRA DE NORMAS TÉCNICAS, 1985).

Conforme mencionado, apenas o valor do nível de referência de campo elétrico é recomendado na faixa de passagem. Assim, foi preciso criar a NBR 15415, que especifica os 
RESENDE, P. C.; CAMPOS, G. L. Estudo sobre as principais regulamentações dos campos eletromagnéticos em linhas de transmissão operando em regime permanente

níveis eletromagnéticos de medição e exposição para instalações de geração, transmissão, distribuição e energia elétrica acima de $1 \mathrm{kV}$ operando na frequência de $50 \mathrm{~Hz}$ a $60 \mathrm{~Hz}$ e será apresentado a seguir neste texto (ASSOCIAÇÃO BRASILEIRA DE NORMAS TÉCNICAS, 2006).

\subsubsection{Métodos de Medições e Níveis de Referência para Exposição a Campos Elétricos e Magnéticos na Frequência de $50 \mathrm{~Hz}$ e $60 \mathrm{~Hz}$ - NBR 15415}

A NBR 15415 de outubro de 2006, foi criada a devido ao desenvolvimento e comercialização de medidores de campo e ao aumento do interesse em caracterizar a exposição humana a campos elétricos e magnéticos quase-estáticos em vários ambientes. Campos quase-estáticos incluem dispositivos que operam na frequência de $50 \mathrm{~Hz}$ e $60 \mathrm{~Hz}$, ou seja, que operam em regime permanente (VIEIRA, 2013), e produzem campos e harmônicas na frequência industrial.

A NBR 15415 estabelece a metodologia de medição e níveis de referência para exposição a campos elétricos e magnéticos de $50 \mathrm{~Hz}$ e $60 \mathrm{~Hz}$ para público geral, ao redor de instalações de geração, transmissão e distribuição de energia elétrica acima de $1 \mathrm{kV}$. No entanto, os valores de referência para população ocupacional nos ambientes de trabalho são definidos por normas regulamentadores do Ministério do Trabalho. Os limites de magnitude de campos magnéticos e elétricos abordados por essa norma são de 100 nT a 100 mT e $1 \mathrm{~V} / \mathrm{m}$ a $50 \mathrm{kV} / \mathrm{m}$, respectivamente.

Os objetivos da NBR 15415 estão descritos abaixo:

a) definir algumas terminologias importantes;

b) identificar os requisitos de especificação do medidor de campo;

c) especificar os requisitos aplicáveis à incerteza da instrumentação;

d) especificar as características gerais dos campos;

e) especificar os princípios operacionais da instrumentação;

f) especificar os métodos de medição.

Com respeito à medição de campo, essa norma considera apenas a medição da intensidade do campo elétrico não perturbado em um ponto no espaço.

Alguns critérios são estabelecidos para determinação dos valores de referência. Os valores de campos elétricos e magnéticos reconhecidos pela NBR 15415 foram estabelecidos 
RESENDE, P. C.; CAMPOS, G. L. Estudo sobre as principais regulamentações dos campos eletromagnéticos em linhas de transmissão operando em regime permanente

pela Comissão Internacional para Proteção contra Radiações Não-Ionizadas (ICNIRP) e são recomendadas pela Organização Mundial de Saúde (OMS).

A indução de corrente elétrica é o principal mecanismo de interação dos campos elétricos e magnéticos com as células. Durante o período de exposição, ocorrem os efeitos biológicos que são de caráter imediato, mesmo com exposição a curto prazo. Para exposição a campos magnéticos, os efeitos observados são: estimulação das células nervosas do cérebro, nervos periféricos, músculos e do coração. Já os campos elétricos, podem causar choques e queimaduras pelo contato com objetos condutores, e como consequência, dificuldades de respiração e fibrilação ventricular.

Por isso, o nível de restrição básica para exposição humana a campos elétricos e magnéticos é estabelecido a partir de correlações entre grandezas físicas e seus efeitos biológicos da exposição. Para teoria de campo, é mais interessante utilizar o conceito de densidade de corrente, pois os eventos de corrente ocorrem em um ponto e não em uma região ampla (HAYT; BUCK, 2012). A densidade de corrente elétrica é a grandeza utilizada para especificar essas correlações, pois o corpo humano na presença de campos elétricos e magnéticos está sujeito a tensões e correntes induzidas. Como referência, o valor de densidade elétrica de $100 \mathrm{~mA} / \mathrm{m}^{2}$ foi estabelecido.

Os campos elétricos estão associados à presença de cargas elétricas e os campos magnéticos ao movimento físico dessas cargas, nas baixas frequências. Como pode-se decompor, a interferência eletromagnética em acoplamento elétrico (capacitivo e condutivo) e magnético (indutivo) (VIEIRA, 2013), os níveis de referência para campos elétricos e magnéticos devem ser considerados separadamente, calculados através de modelos matemáticos adequados.

A partir dos critérios estabelecidos, os níveis de referência para o público geral correspondem a campos com densidades de correntes inferiores a $2 \mathrm{~mA} / \mathrm{m}^{2}$. A Tabela 6 mostra os níveis de referência para campo elétrico e magnético, no limite da faixa de segurança de uma linha de transmissão, no lado externo do perímetro da subestação ou usina e no limite do afastamento mínimo do circuito de distribuição. 
RESENDE, P. C.; CAMPOS, G. L. Estudo sobre as principais regulamentações dos campos eletromagnéticos em linhas de transmissão operando em regime permanente

Tabela 6 - Níveis de referência em valor eficaz de exposição a campos elétricos e magnéticos

\begin{tabular}{ccccc}
\hline Frequência & \multicolumn{2}{c}{$\mathbf{5 0 ~ H z}$} & \multicolumn{2}{c}{$\mathbf{6 0 ~ H z}$} \\
\hline \multirow{2}{*}{ Campo } & Elétrico & Magnético & Elétrico & Magnético $\mu \mathrm{T}$ \\
& $\mathrm{kV} / \mathrm{m}$ & $\mu \mathrm{T}$ & $\mathrm{kV} / \mathrm{m}$ & \\
\multirow{2}{*}{ Público em Geral } & 5,00 & 100,00 & 4,16 & 83,33 \\
\hline
\end{tabular}

Fonte: Associação Brasileira de Normas Técnicas (2006).

A norma estabelece algumas especificações de instrumentos para realizar as medições de campos elétricos e magnéticos alternados, a calibração desses instrumentos, a normatização para cômputo das incertezas nas medições e as etapas de registro e referência dos resultados medidos.

No procedimento de medição de campo elétrico, deve ser realizada pelo menos uma medição na instalação. No procedimento de medição de campo magnético, deve ser realizada uma medição de densidade de fluxo magnético da instalação, preferencialmente no período de maior carregamento. No caso de várias medições, adotar o maior valor. Para linhas de transmissão, a medição deve ser no limite da faixa de segurança a uma altura de 1,5 $\mathrm{m}$ do solo, transversalmente ao eixo da linha, no ponto de menos distância entre o condutor da fase e o solo texto (ASSOCIAÇÃO BRASILEIRA DE NORMAS TÉCNICAS, 2006).

Como as normas passam por atualizações e alterações, a Lei Federal 11.934 de maio de 2009 e a Resolução Normativa 398 de março de 2010 da Agência Nacional de Energia Elétrica (ANEEL) vieram para complementar a NBR 15415, conforme será apresentado no próximo item deste trabalho.

\subsubsection{Lei Federal 11.934/09 e Resolução Normativa 398 da ANEEL}

A Lei Federal 11.934 de 5 de maio de 2009 estabelece limites à exposição humana a campos elétricos, magnéticos e eletromagnéticos, associados ao funcionamento de estações transmissoras de radiocomunicação, de terminais de usuário e de sistemas de energia elétrica nas faixas de frequências até $300 \mathrm{GHz}$. Diferente da NBR 15415, que determina níveis de referência somente para público geral, a Lei Federal define limites para os trabalhadores, ou seja, público ocupacional, que estão expostos a esses campos. 
RESENDE, P. C.; CAMPOS, G. L. Estudo sobre as principais regulamentações dos campos eletromagnéticos em linhas de transmissão operando em regime permanente

Tal como a NBR 15415, a Lei Federal adota os limites reconhecidos pela OMS, que são determinados pela ICNIRP, para exposição ocupacional e público geral.

Segundo a Lei Federal, o órgão regulador federal de serviços de energia elétrica, a ANEEL, é responsável pela fiscalização do atendimento aos limites de campos estabelecidos.

Cabe ao órgão regulador adotar algumas providências como:

a) editar a regulamentação sobre os métodos de avaliação e procedimentos necessários para a verificação do nível de campo elétrico e magnético;

b) tornar as informações públicas e banco de dados sobre as medições de campos elétricos e magnéticos realizadas, gerados por sistemas de transmissão de energia elétrica para acompanhamento dos níveis de exposição no território nacional;

c) solicitar medição ou verificação de campos, para autorização de operação de novo sistema de transmissão de energia elétrica a ser integrado à Rede Básica Nacional.

Sempre que houver a instalação de novo sistema de transmissão de energia ou alteração nas características vigentes do sistema de transmissão, as concessionárias de transmissão de energia elétrica deverão realizar medições dos níveis de campo elétrico e magnético ou apresentar os cálculos efetuados com a metodologia válida (BRASIL, 2009).

A ANEEL criou a Resolução Normativa 398 de 23 de março de 2010 para regulamentar a Lei Federal 11.934/09. Deste modo, os níveis de referência para campos elétricos e magnéticos variantes no tempo na frequência de $60 \mathrm{~Hz}$ são apresentados na Tabela 7. Importante destacar que as diferenças dos valores da Tabela 6, da NBR 15415, com os valores da Tabela 7 são devidas às referências utilizadas pelas regulamentações. A NBR 15415 utiliza a ICNIRP de 1990, já a ANEEL usa como referência ICNIRP de 2010.

Tabela 7 - Níveis de referência em valor eficaz de exposição a campos elétricos e magnéticos

\begin{tabular}{ccccc}
\hline Frequência & \multicolumn{2}{c}{$\mathbf{5 0 ~ H z}$} & \multicolumn{2}{c}{$\mathbf{6 0 ~ H z}$} \\
\hline Campo & Elétrico & Magnético & Elétrico & Magnético $\mu \mathrm{T}$ \\
& $\mathrm{kV} / \mathrm{m}$ & $\mu \mathrm{T}$ & $\mathrm{kV} / \mathrm{m}$ & \\
Público em Geral & 5,00 & 200,00 & 4,17 & 200,00 \\
Público Ocupacional & 10,00 & 1000,00 & 8,33 & 1000,00 \\
\hline
\end{tabular}

Fonte: Agência Nacional de Energia Elétrica (2010).

Os agentes de transmissão devem, até o final da fase de comissionamento de novos empreendimentos, adicionar à documentação exigida pela ANEEL o memorial de cálculo ou 
RESENDE, P. C.; CAMPOS, G. L. Estudo sobre as principais regulamentações dos campos eletromagnéticos em linhas de transmissão operando em regime permanente

o relatório das medições dos campos elétricos e magnéticos. As medições devem condizer com a metodologia estabelecida pela NBR 15415/2006, no período de carga pesada (AGÊNCIA NACIONAL DE ENERGIA ELÉTRICA, 2010).

\title{
5 CONCLUSÃO
}

O prévio estudo das principais regulamentações internacionais foi importante para conhecer e entender a origem dos valores apresentados nas normas regulamentadoras brasileiras. As regulamentações internacionais são estudadas por vários anos e atualizadas constantemente.

Porém, apesar da grande extensão do território brasileiro e por consequência, um vasto sistema de transmissão de energia elétrica, as regulamentações brasileiras acerca dos campos eletromagnéticos são recentes.

Destaca-se que cada um dos documentos nacionais apresentados neste trabalho veio para preencher uma lacuna ou inconsistência do documento anterior. Por isso, há diferenças de valores entre um documento e outro. Assim, a evolução do tema, as preocupações referentes à saúde e desempenho dos dispositivos próximos às linhas de transmissão fazem com que pesquisas sobre o assunto sejam cada vez mais frequentes, implicando na constante revisão de normas regulamentadoras pelos órgãos responsáveis.

Contudo, a sociedade vem conhecendo cada vez mais sobre o assunto e procurando novas informações, apesar dos estudos sobre efeitos à saúde dos campos eletromagnéticos não terem resultados definitivos.

\section{STUDY ABOUT MAIN REGULATIONS OF ELECTROMAGNETIC FIELDS IN TRANSMISSION LINES OPERATING ON STEADY STATE}

\begin{abstract}
This article aims to present, in a general way, the main national and international regulations and recommendations, regarding levels of human exposure limit to electric and magnetic fields for transmission lines, operating in the frequency of $50-60 \mathrm{~Hz}$. a survey is carried out to identify the main national and international institutions accredited to elaborate the regulations. After this study, abstracts are made of the regulatory norms on electromagnetic fields of each institution, in order to facilitate the reader's understanding and to show in a simplified way the limits of electric and magnetic fields established by the institutions.
\end{abstract}

Keywords: Transmission lines. Electric field. Magnetic field. Regulation. Reference level. 
RESENDE, P. C.; CAMPOS, G. L. Estudo sobre as principais regulamentações dos campos eletromagnéticos em linhas de transmissão operando em regime permanente

\section{REFERÊNCIAS}

ASSOCIAÇÃO BRASILEIRA DE NORMAS TÉCNICAS. NBR 5422: projetos de linhas de transmissão de energia elétrica. Rio de Janeiro: ABNT, 1985.

NBR 15415: métodos de medição e níveis de referência para exposição a campos elétricos e magnéticos na frequência de $50 \mathrm{~Hz}$ e $60 \mathrm{~Hz}$. Rio de Janeiro: ABNT, 2006.

AGÊNCIA NACIONAL DE ENERGIA ELÉTRICA. Resolução Normativa n 394, de 23 de março de 2010. Brasília, 2010.

BECKER, W. D. Estudo de técnicas para redução de campos magnéticos gerados por linhas de transmissão de potência. 2008. 95 f. Dissertação (Mestrado em Engenharia Elétrica), Universidade Federal de Santa Catarina, Florianópolis, 2008.

BRASIL. Lei ${ }^{\circ} 11.934$, de 5 de maio de 2009. Dispõe sobre limites à exposição humana a campos elétricos, magnéticos e eletromagnéticos. Diário Oficial [da] República Federativa do Brasil. Brasília, 5 maio 2009.

CAMPOS, O. L. Estudo de caso sobre impactos ambientais de linhas de transmissão na Região Amazônica, BNDES Setorial, n. 32, p. 231-266, set. 2010.

COSTA, L. A. MONTEIRO, V. B. Minimização do campo magnético em linhas de transmissão. Disponível em: <ftp://labattmot.ele.ita.br/ele/santos_dias/Leitura/t3.pdf> Acesso em: 24 de abril de 2016.

THE COUNCIL OF THE EUROPEAN UNION. Council recommendation of 12 July 1999 on the limitation of exposure of the general public to electromagnetic fields ( $0 \mathrm{~Hz}$ to 300 GHz): (1999/519/EC). Official Journal of the European Communities, v. 59, p. 59-70, 1999.

EUROPEAN UNION. The European Council. Bruxelas, 2016. Disponível em: <http://www.consilium.europa.eu/>. Acesso em: 25 abr. 2016.

HAYT, W. H.; BUCK, J. A. Eletromagnetismo. 8. ed. Rio Grande do Sul: McGraw-Hill, 2012.

INTERNATIONAL COMMISSION ON NON-IONIZING RADIATION PROTECTION. Guidelines for limiting exposure to time-varying electric and magnetic fields ( $1 \mathrm{~Hz}$ to 100 kHz), Health Physics, v. 99, n. 6, p. 818-836, dez. 2010.

INSTITUTE OF ELECTRIC AND ELECTRONIC ENGINEERS - INTERNACIONAL COMMITTEE ON ELECTROMAGNETIC SAFETY. IEEE Standard for Safety Levels 
RESENDE, P. C.; CAMPOS, G. L. Estudo sobre as principais regulamentações dos campos eletromagnéticos em linhas de transmissão operando em regime permanente

with Respect to Human Exposure to Electromagnetic Fields, 3kHz to $300 \mathrm{GHz}$. New York, 2006. Disponível em: 〈http://emfguide.itu.int/pdfs/C95.1-2005.pdf〉. Acesso em: 25 abr. 2016.

INSTITUTE OF ELECTRIC AND ELECTRONIC ENGINEERS - ADVANCING TECHNOLOGY FOR HUMANITY. Mission and vision. [New York, 20--]. Disponível em: <https://www.ieee.org/about/vision_mission.html>. Acesso em: 25 abr. 2016.

SOUZA, C. J. Determinação da largura de faixa de segurança de linhas de transmissão: um estudo paramétrico. 2012. 85 f. Dissertação (Mestrado em Engenharia Elétrica) Universidade Federal de Minas Gerais, Belo Horizonte, 2012.

VIEIRA, H. R. Acoplamento magnético entre linhas de transmissão operando em regime permanente e dutos metálicos aéreos. 2013. 158 f. Dissertação (Mestrado em Engenharia Elétrica) - Universidade Federal de São João del-Rei, São João Del Rei, 2013.

Recebido em: 10/10/2016

Aprovado em: 29/08/2017

Publicado em: 23/11/2017 